Journal of Mathematics and Informatics

Vol. 10, 2017, 11-19

ISSN: 2349-0632 (P), 2349-0640 (online)

Published 11 December 2017

www.researchmathsci.org

DOI: http://dx.doi.org/10.22457/jmi.v10a2

Journal of

Mathematics and

Informatics

\title{
On the Ternary Quadratic Diophantine Equation $3\left(X^{2}+Y^{2}\right)-5 X Y=75 Z^{2}$
}

\section{S.Thenmozhi ${ }^{1}$ and S.Vidhyalakshmi ${ }^{2}$}

Department of mathematics, SIGC, Trichy-2, TamilNadu, INDIA.

'e-mail: sthenmozhi720@gmail.com; ${ }^{2}$ e-mail: vidhyasigc@ gmail.com

Received 15 November 2017; accepted 4 December 2017

Abstract. The ternary quadratic equation given by $3\left(X^{2}+Y^{2}\right)-5 X Y=75 Z^{2}$ is considered and searched for its many different integer solutions. Four different choices of integer solutions to the above equation are presented. A few interesting relations between the solutions and special polygonal numbers are presented.

Keywords: Ternary quadratic, integer solutions.

AMS Mathematics Subject Classification (2010): $11 \mathrm{D} 09$

\section{Introduction}

The Diophantine equations offer an unlimited field for research due to their variety (1-3). In particular, one may refer (4-15) for quadratic equations with three unknowns.

This communication concerns with yet another interesting equation $3\left(X^{2}+Y^{2}\right)-5 X Y=75 Z^{2}$ representing non-homogeneous quadratic equation with three unknowns for determining its infinitely many non-zero integral points. Also, few interesting relations among the solutions are presented.

\section{Notations}

- $t_{m, n}=n^{\text {th }}$ term of a regular polygon with $m$ sides

$$
=n\left(1+\frac{(n-1)(m-2)}{2}\right)
$$

- Triangular number of rank $\mathrm{n}, T_{3, n}=\frac{n(n+1)}{2}$

\section{Method of analysis}

The ternary quadratic Diophantine equation to be solved for its non-zero distinct integral solution is

$$
3\left(X^{2}+Y^{2}\right)-5 X Y=75 Z^{2}
$$

The solution of linear transformations $(u \neq v \neq 0)$ 


\section{S.Thenmozhi and S.Vidhyalakshmi}

$X=u+v, Y=u-v$

In (1) leads to

$u^{2}+11 v^{2}=75 Z^{2}$

Different patterns of solutions of (1) are presented below:

\subsection{PATTERN-1}

Write 75 as

$75=(8+i \sqrt{11})(8-i \sqrt{11})$

Assume $Z=a^{2}+11 b^{2}$

Where $\mathrm{a}, \mathrm{b}$ are non-zero distinct integers.

Use (4) and (5) in (3), we get

$u^{2}+11 v^{2}=(8+i \sqrt{11})(8-i \sqrt{11})\left(a^{2}+11 b^{2}\right)$

Equating the positive and negative factors, we get

$u+i \sqrt{11} v=(8+i \sqrt{11})(a+i \sqrt{11} b)^{2}$

$u-i \sqrt{11} v=(8-i \sqrt{11})(a-i \sqrt{11} b)^{2}$

Equating real and imaginary parts either in $(5 a)$ or $(5 b)$ we get

$u=u(a, b)=8 a^{2}-88 b^{2}-22 a b$

$v=v(a, b)=a^{2}-11 b^{2}+16 a b$

Substituting the values of $\mathrm{u}$ and $\mathrm{v}$ in (2) we get

$$
\begin{aligned}
& X=X(a, b)=9 a^{2}-99 b^{2}-6 a b \\
& Y=Y(a, b)=7 a^{2}-77 b^{2}-38 a b
\end{aligned}
$$

Thus (6), (7) and (5) represents non-zero distinct integral solutions of (1) in two parameters.

Properties observed are as follows:

1. $X(a, 1)-t_{20, a}+99 \equiv 0(\bmod 2)$

2. $7 Z(a, 1)+Y(a, 1)-t_{30, a} \equiv 0(\bmod 5)$

3. $9 Z(a, 1)+X(a, 1)-t_{38, a} \equiv 0(\bmod 11)$

4. $6\left(t_{30, \alpha^{2}}-7 Z\left(\alpha^{2}, 1\right)-Y\left(\alpha^{2}, 1\right)\right)=6 * 5\left(\alpha^{2}\right)$ is a nasty number

5. $Z(a, a+1)-t_{26, a}-11 \equiv 0(\bmod 3)$

6. $Z(a, a+2)-t_{26, a}-44 \equiv 0(\bmod 5)$

\subsection{PATTERN-2}

Write 75 as

$75=\frac{(17+i \sqrt{11})(17-i \sqrt{11})}{4}$ 
On The Ternary Quadratic Diophantine Equation $3\left(X^{2}+Y^{2}\right)-5 X Y=75 Z^{2}$

Assume $Z=a^{2}+11 b^{2}$

where $\mathrm{a}, \mathrm{b}$ are non-zero distinct integers.

Use (8) and (5) in (3), we get

$u^{2}+11 v^{2}=\frac{(17+i \sqrt{11})(17-i \sqrt{11})}{4}\left(a^{2}+11 b^{2}\right)$

Equating the positive and negative factors, we get

$$
\begin{aligned}
& u+i \sqrt{11} v=\left(\frac{17+i \sqrt{11}}{2}\right)(a+i \sqrt{11} b)^{2} \\
& u-i \sqrt{11} v=\left(\frac{17-i \sqrt{11}}{2}\right)(a-i \sqrt{11} b)^{2}
\end{aligned}
$$

Equating real and imaginary parts either in (9a) or (9b) we get

$$
\begin{aligned}
& u=u(a, b)=1 / 2\left\{17 a^{2}-187 b^{2}-22 a b\right\} \\
& v=v(a, b)=1 / 2\left\{a^{2}-11 b^{2}+34 a b\right\}
\end{aligned}
$$

Substituting the values of $\mathrm{u}$ and $\mathrm{v}$ in (2) we get

$$
\begin{aligned}
& X=X(a, b)=9 a^{2}-99 b^{2}+6 a b \\
& Y=Y(a, b)=8 a^{2}-88 b^{2}-28 a b
\end{aligned}
$$

Thus (10), (11) and (5) represents non-zero distinct integral solutions of (1) in two parameters.

Properties observed are as follows:
1. $X(a, 1)-t_{20, a}+99 \equiv 0(\bmod 14)$
2. $8 Z(a, 1)+Y(a, 1)-t_{34, a} \equiv 0(\bmod 13)$
3. $9 Z(a, 1)+X(a, 1)-t_{38, a} \equiv 0(\bmod 23)$

\subsection{PATTERN-3}

(3) is written as

$$
\begin{aligned}
& u^{2}+11 v^{2}=64 Z^{2}+11 Z^{2} \\
& u^{2}-64 Z^{2}=11\left(Z^{2}-v^{2}\right) \\
& (u+8 Z)(u-8 Z)=11(Z+v)(Z-v)
\end{aligned}
$$

Write (12) as,

$$
\frac{u+8 Z}{Z+v}=\frac{11(Z-v)}{u-8 Z}=\frac{\alpha}{\beta}, \beta \neq 0
$$

we have $\frac{u+8 Z}{Z+v}=\frac{\alpha}{\beta} \quad \Rightarrow u \beta+v \alpha+(8 \beta-\alpha) Z=0$

From the $1^{\text {st }}$ and $3^{\text {rd }}$ factors of (12a), we get

$$
\frac{11(Z-v)}{u-8 Z}=\frac{\alpha}{\beta} \Rightarrow u \alpha+11 v \beta-(8 \alpha+11 \beta) Z=0
$$

Applying the method of cross multiplication for solving (13) and (14), we have 


$$
\begin{aligned}
& u=u(\alpha, \beta)=8 \alpha^{2}-88 \beta^{2}+22 \alpha \beta \\
& v=v(\alpha, \beta)=11 \beta^{2}-\alpha^{2}+16 \alpha \beta \\
& Z=Z(\alpha, \beta)=\alpha^{2}+11 \beta^{2}
\end{aligned}
$$

Substituting the values of $\mathrm{u}$ and $\mathrm{v}$ in (2)

$$
\begin{aligned}
& X=X(\alpha, \beta)=7 \alpha^{2}-77 \beta^{2}+38 \alpha \beta \\
& Y=Y(\alpha, \beta)=9 \alpha^{2}-99 \beta^{2}+6 \alpha \beta \\
& Z=Z(\alpha, \beta)=\alpha^{2}+11 \beta^{2}
\end{aligned}
$$

Properties observed are as follows:
1. $X(\alpha, 1)-t_{16, \alpha}+77 \equiv 0(\bmod 11)$
2. $9 Z(\alpha, 1)+Y(\alpha, 1)-t_{38, \alpha} \equiv 0(\bmod 23)$
3. $7 Z(\alpha, 1)+X(\alpha, 1)-t_{30, \alpha} \equiv 0(\bmod 51)$
4. $Y(\alpha, 1)-t_{20, \alpha}+99 \equiv 0(\bmod 2)$

\subsection{PATTERN-4}

Write (12) as,

$$
\frac{u+8 Z}{11(Z+v)}=\frac{Z-v}{u-8 Z}=\frac{\alpha}{\beta}, \beta \neq 0
$$

From the $1^{\text {st }}$ and $2^{\text {nd }}$ factors of $(12 b)$, we get

$$
\frac{u+8 Z}{11(Z+v)}=\frac{\alpha}{\beta} \Rightarrow u \beta-11 v \alpha-(11 \alpha-8 \beta) Z=0
$$

From the $1^{\text {st }}$ and $3^{\text {rd }}$ factors of $(12 \mathrm{~b})$, we get

$$
\frac{Z-v}{u-8 Z}=\frac{\alpha}{\beta} \quad \Rightarrow u \alpha+v \beta-(8 \alpha+\beta) Z=0
$$

Applying the method of cross multiplication for solving (15) and (16), we have

$$
\begin{aligned}
& u=u(\alpha, \beta)=88 \alpha^{2}-8 \beta^{2}+22 \alpha \beta \\
& v=v(\alpha, \beta)=\beta^{2}-11 \alpha^{2}+16 \alpha \beta \\
& Z=Z(\alpha, \beta)=11 \alpha^{2}+\beta^{2}
\end{aligned}
$$

Substituting the values of $\mathrm{u}$ and $\mathrm{v}$ in (2)

$$
\begin{aligned}
& X=X(\alpha, \beta)=77 \alpha^{2}-7 \beta^{2}+38 \alpha \beta \\
& Y=Y(\alpha, \beta)=99 \alpha^{2}-9 \beta^{2}+6 \alpha \beta \\
& Z=Z(\alpha, \beta)=11 \alpha^{2}+\beta^{2}
\end{aligned}
$$

Properties observed are as follows:

1. $X(\alpha, 1)-t_{156, \alpha}-7 \equiv 0(\bmod 6)$

2. $15\left\lfloor 7 Z(\alpha, 1)+X(\alpha, 1)-t_{60, \alpha}-t_{52, \alpha}-180 t_{3, \alpha}\right\rfloor$ is nasty number 
On The Ternary Quadratic Diophantine Equation $3\left(X^{2}+Y^{2}\right)-5 X Y=75 Z^{2}$

3. $2\left\lfloor 9 Z(\alpha, 1)+Y(\alpha, 1)-12 t_{3, \alpha}\right\rfloor$ is a nasty number

4. $Z(\alpha, \alpha+1)-t_{26, \alpha}-1 \equiv 0(\bmod 13)$

\section{Remarkable observations}

\subsection{Triple 1}

Let

$$
\begin{aligned}
& u_{1}=u_{0}+9 h \\
& v_{1}=v_{0} \\
& Z_{1}=Z_{0}+h
\end{aligned}
$$

Substituting in (3) we get

$u_{1}^{2}+11 v_{1}^{2}=75 Z_{1}^{2}$

$\left(u_{0}+9 h\right)^{2}+11 v_{0}^{2}=75\left(Z_{0}+h\right)^{2}$

$h=25 Z_{0}-3 u_{0}$

Substituting $h$ value in (17) we get

$$
\begin{aligned}
& u_{1}=225 Z_{0}-26 u_{0} \\
& v_{1}=v_{0} \\
& Z_{1}=26 Z_{0}-3 u_{0}
\end{aligned}
$$

Hence the matrix representation of the above solution is

$$
\left[\begin{array}{l}
u_{1} \\
Z_{1}
\end{array}\right]=\left[\begin{array}{cc}
-26 & 225 \\
-3 & 26
\end{array}\right]\left[\begin{array}{l}
u_{0} \\
Z_{0}
\end{array}\right]
$$

Similarly, to find the $\mathrm{n}^{\text {th }}$ solution of (3)

let $A=\left[\begin{array}{cc}-26 & 225 \\ -3 & 26\end{array}\right]$

To find eigen values of $\mathrm{A}$.

Consider $|A-\lambda I|=0$

$\left|\begin{array}{cc}-26-\lambda & 225 \\ -3 & 26-\lambda\end{array}\right|=0 \Rightarrow(-26-\lambda)(26-\lambda)+675=0$

$\lambda= \pm 1$

The eigen values are $\alpha=1$ and $\beta=-1$

Substituting $\alpha, \beta$ and $A$ values in the below equation

$$
M^{n}=\frac{\alpha^{n}}{(\alpha-\beta)}(A-\beta I)+\frac{\beta^{n}}{(\beta-\alpha)}(A-\alpha I)
$$

We get

$$
M^{n}=\frac{1}{2}\left(\begin{array}{cc}
-25 & 225 \\
-3 & 27
\end{array}\right)+\frac{(-1)^{n}}{2}\left(\begin{array}{cc}
27 & -225 \\
3 & -25
\end{array}\right)
$$


S.Thenmozhi and S.Vidhyalakshmi

$\therefore\left[\begin{array}{l}u_{n} \\ Z_{n}\end{array}\right]=\frac{1}{2}\left(\begin{array}{cc}-25+(-1)^{n} 27 & 225-225(-1)^{n} \\ -3+(-1)^{n} 3 & 27-(-1)^{n} 25\end{array}\right)\left[\begin{array}{l}u_{0} \\ Z_{0}\end{array}\right]$

Then we get $\mathrm{n}^{\text {th }}$ solution,

$$
\begin{aligned}
& X_{n}=u_{n}+v_{n}=\frac{1}{2}\left\{\left(-25+(-1)^{n} 27\right) u_{0}+\left(225-(-1)^{n} 225\right) Z_{0}\right\}+v_{0} \\
& Y_{n}=u_{n}-v_{n}=\frac{1}{2}\left\{\left(-25+(-1)^{n} 27\right) u_{0}+\left(225-(-1)^{n} 225\right) Z_{0}\right\}-v_{0} \\
& Z_{n}=\frac{1}{2}\left\{\left(-3+(-1)^{n} 3\right) u_{0}+\left(27-(-1)^{n} 25\right) Z_{0}\right\}
\end{aligned}
$$

\subsection{Triple 2}

Let

$$
\begin{aligned}
& u_{1}=8 u_{0} \\
& v_{1}=-8 v_{0}+3 h \\
& Z_{1}=8 Z_{0}+h
\end{aligned}
$$

Substituting in (3) we get

$u_{1}^{2}+11 v_{1}^{2}=75 Z_{1}^{2}$

$\left(8 u_{0}\right)^{2}+11\left(-8 v_{0}+3 h\right)^{2}=75\left(8 Z_{0}+h\right)^{2}$

$h=50 Z_{0}+22 v_{0}$

Substituting h value in (18) we get

$$
\begin{aligned}
& v_{1}=58 v_{0}+150 Z_{0} \\
& Z_{1}=22 v_{0}+58 Z_{0}
\end{aligned}
$$

Hence the matrix representation of the above solution is

$$
\left[\begin{array}{l}
v_{1} \\
Z_{1}
\end{array}\right]=\left[\begin{array}{cc}
58 & 150 \\
22 & 58
\end{array}\right]\left[\begin{array}{l}
v_{0} \\
Z_{0}
\end{array}\right]
$$

Similarly, to find the $\mathrm{n}^{\text {th }}$ solution of (3)

let $A=\left[\begin{array}{cc}58 & 150 \\ 22 & 58\end{array}\right]$

To find eigen values of $A$.

Consider $|A-\lambda I|=0$

$$
\begin{aligned}
& \left|\begin{array}{cc}
58-\lambda & 150 \\
22 & 58-\lambda
\end{array}\right|=0 \Rightarrow(58-\lambda)(58-\lambda)-3300=0 \\
& \lambda=58 \pm 10 \sqrt{33}
\end{aligned}
$$

The eigen values are $\alpha=58+10 \sqrt{33}$ and $\beta=58-10 \sqrt{33}$ 
On The Ternary Quadratic Diophantine Equation $3\left(X^{2}+Y^{2}\right)-5 X Y=75 Z^{2}$

$$
\begin{aligned}
& \therefore M^{n}=\frac{(58+10 \sqrt{33})^{n}}{20 \sqrt{33}}\left(\begin{array}{cc}
10 \sqrt{33} & 150 \\
22 & 10 \sqrt{33}
\end{array}\right)+\frac{(58-10 \sqrt{33})^{n}}{20 \sqrt{33}}\left(\begin{array}{cc}
10 \sqrt{33} & -150 \\
-22 & 10 \sqrt{33}
\end{array}\right) \\
& =\frac{1}{20 \sqrt{33}}\left(\begin{array}{cc}
10 \sqrt{33} A_{n} & 150 B_{n} \\
22 B_{n} & 10 \sqrt{33} A_{n}
\end{array}\right) \\
& \text { where } A_{n}=\left[(58+10 \sqrt{33})^{n}+(58-10 \sqrt{33})^{n}\right] \\
& B_{n}=\left[(58+10 \sqrt{33})^{n}-(58-10 \sqrt{33})^{n}\right]
\end{aligned}
$$

Thus,

$$
\left[\begin{array}{l}
v_{n} \\
Z_{n}
\end{array}\right]=\frac{1}{20 \sqrt{33}}\left(\begin{array}{cc}
10 \sqrt{33} A_{n} & 150 B_{n} \\
22 B_{n} & 10 \sqrt{33} A_{n}
\end{array}\right)\left[\begin{array}{l}
v_{0} \\
Z_{0}
\end{array}\right]
$$

Then we get $\mathrm{n}^{\text {th }}$ solution,

$$
\begin{aligned}
& X_{n}=u_{n}+v_{n}=8^{n} u_{0}+\frac{1}{20 \sqrt{33}}\left\{10 \sqrt{33} A_{n} v_{0}+150 B_{n} Z_{0}\right\} \\
& Y_{n}=u_{n}-v_{n}=8^{n} u_{0}-\frac{1}{20 \sqrt{33}}\left\{10 \sqrt{33} A_{n} v_{0}+150 B_{n} Z_{0}\right\} \\
& Z_{n}=\frac{1}{20 \sqrt{33}}\left\{22 B_{n} v_{0}+10 \sqrt{33} A_{n} Z_{0}\right\}
\end{aligned}
$$

\subsection{Triple 3}

Let

$$
\begin{aligned}
& u_{1}=h-6 u_{0} \\
& v_{1}=h-6 v_{0} \\
& Z_{1}=6 Z_{0}
\end{aligned}
$$

Substituting in (3) we get

$$
\begin{aligned}
& u_{1}^{2}+11 v_{1}^{2}=75 Z_{1}^{2} \\
& \left(h-6 u_{0}\right)^{2}+11\left(h-6 v_{0}\right)^{2}=75\left(6 Z_{0}\right)^{2} \\
& h=u_{0}+11 v_{0}
\end{aligned}
$$

Substituting h value in (19) we get

$$
\begin{aligned}
& u_{1}=-5 u_{0}+11 v_{0} \\
& v_{1}=u_{0}+5 v_{0}
\end{aligned}
$$

Hence the matrix representation of the above solution is

$$
\left[\begin{array}{l}
u_{1} \\
v_{1}
\end{array}\right]=\left[\begin{array}{cc}
-5 & 11 \\
1 & 5
\end{array}\right]\left[\begin{array}{l}
u_{0} \\
v_{0}
\end{array}\right]
$$

Similarly, to find the $\mathrm{n}^{\text {th }}$ solution of (3) 


\section{S.Thenmozhi and S.Vidhyalakshmi}

let $A=\left[\begin{array}{cc}-5 & 11 \\ 1 & 5\end{array}\right]$

To find eigen values of $\mathrm{A}$ :

Consider $|A-\lambda I|=0$

$$
\left|\begin{array}{cc}
-5-\lambda & 11 \\
1 & 5-\lambda
\end{array}\right|=0 \Rightarrow(-5-\lambda)(5-\lambda)+11=0
$$

The eigen values are $\alpha=6$ and $\beta=-6$

$$
\therefore M^{n}=\frac{6^{n}}{12}\left(\begin{array}{ll}
1 & 11 \\
1 & 11
\end{array}\right)+\frac{(-6)^{n}}{12}\left(\begin{array}{cc}
11 & -11 \\
-1 & 1
\end{array}\right)
$$

Then

$$
\left[\begin{array}{l}
u_{n} \\
v_{n}
\end{array}\right]=\frac{1}{12}\left(\begin{array}{cc}
6^{n}+11(-6)^{n} & 11 * 6^{n}-11(-6)^{n} \\
6^{n}-(-6)^{n} & 11 * 6^{n}+(-6)^{n}
\end{array}\right)\left[\begin{array}{c}
u_{0} \\
v_{0}
\end{array}\right]
$$

Then we get $\mathrm{n}^{\text {th }}$ solution,

$$
\begin{aligned}
& X_{n}=u_{n}+v_{n}=\frac{1}{12}\left\{\left(2 * 6^{n}+10(-6)^{n}\right) u_{0}+\left(22 * 6^{n}-10(-6)^{n}\right) v_{0}\right\} \\
& Y_{n}=u_{n}-v_{n}=\frac{1}{12}\left\{\left(2 * 6^{n}+10(-6)^{n}\right) u_{0}-\left(22 * 6^{n}-10(-6)^{n}\right) v_{0}\right\} \\
& Z_{n}=6^{n} Z_{0}
\end{aligned}
$$

\section{Conclusion}

In this paper, we have presented infinitely many non-zero distinct integer solutions to the ternary quadratic equation

$$
3\left(X^{2}+Y^{2}\right)-5 X Y=75 Z^{2}
$$

representing a homogeneous cone. As Diophantine equations are rich in variety.

To conclude, one may search for other forms of three dimensional surfaces, namely, non-homogeneous cone, paraboloid, ellipsoid, hyperboloid, hyperbolic paraboloid and so on for finding integral points on them and corresponding properties

\section{REFERENCES}

1. L.E.Dickson, History of Theory of Numbers and Diophantine Analysis, Vol.2, Dover publications, New York 2005.

2. L.J.Mordell, Diophantine Equations, Academic Press, New York 1970.

3. R.D.Carmicheal, The Theory of Numbers and Diophantine Analysis, Dover publications, New York 1959.

4. M.A.Gopalan and D.Getha, Lattice points on the Hyperboloid of two sheets $X^{2}-6 X Y+Y^{2}+6 X-2 Y+5=Z^{2}+4$, Impact J. Sci. Tech., 4 (2010) 23-32.

5. M.A.Gopalan, S.Vidhyalakshmi and A.Kavitha, Integral points on the Homogeneous cone $Z^{2}=2 X^{2}-7 Y^{2}$, The Diophantus J Math., 1(2) (2012) 127-136. 
On The Ternary Quadratic Diophantine Equation $3\left(X^{2}+Y^{2}\right)-5 X Y=75 Z^{2}$

6. M.A.Gopalan, S.Vidhyalakshmi and G.Sumathi, Lattice points on the Hyperboloid one sheet $4 Z^{2}=2 X^{2}+3 Y^{2}-4$, The Diophantus J Math., 1(2) (2012) 109-115.

7. M.A.Gopalan, S.Vidhyalakshmi and K.Lakshmi, Integral points on the Hyperboloid two sheets $3 Y^{2}=7 X^{2}-Z^{2}+21$, The Diophantus J Math., 1(2) (2012) 99-107.

8. M.A.Gopalan, S.Vidhyalakshmi and S.Mallika, Observations on Hyperboloid of one sheets $X^{2}+2 Y^{2}-Z^{2}=2$, Bessel J. Math., 2(3) (2012) 221-226.

9. M.A.Gopalan, S.Vidhyalakshmi, T.R.Usha Rani and S.Mallika, Integral points on the Homogeneous cone $6 Z^{2}+3 Y^{2}-2 X^{2}=0$, The Impact J. Sci Tech., 6(1) (2012) 713.

10. M.A.Gopalan, S.Vidhyalakshmi and G.Sumathi, Lattice points on the Elliptic paraboloid $Z=9 X^{2}+4 Y^{2}$, Advances in Theoretical and Applied Mathematics, 7(4) (2012) 379-385.

11. M.A.Gopalan, S.Vidhyalakshmi and T.R.Usha Rani, Integral points on the nonhomogeneous cone $2 Z^{2}+4 X Y+8 X-4 Z=0$, Global Journal of Mathematics and Mathematical Science, 2(1) (2012) 61-67.

12. MA.Gopalan, S.Vidhyalakshmi and K.Lakshmi, Lattice points on the Elliptic paraboloid $16 Y^{2}+9 Z^{2}=4 X$, Bessel J. of Math., 3(2) (2013) 137-145.

13. K.Meena, S.Vidhyalakshmi, E.Bhuvaneswari and R.Presenna, On ternary quadratic diophantine equation $5\left(X^{2}+Y^{2}\right)-6 X Y=20 Z^{2}$, International Journal of Advanced Scientific Research, 1(2) (2016) 59-61.

14. M.A.Gopalan, S.Vidhyalakshmi and U.K.Rajalakshmi, On ternary quadratic Diophantine equation $5\left(X^{2}+Y^{2}\right)-6 X Y=196 Z^{2}$, Journal of Mathematics, 3(5) (2017) 1-10.

15. M.A.Gopalan, S.Vidhyalakshmi and S.Aarthy Thangam, On ternary quadratic equation $X(X+Y)=Z+20$, IJIRSET, 6(8) (2017) 15739-15741. 